Braz J Med Biol Res, September 2011, Volume 44(9) 814-826

doi: 10.1590/S0100-879X2011007500055

Effect of gender on training-induced vascular remodeling in SHR

S.L. Amaral and L.C. Michelin

The Brazilian Journal of Medical and Biological Research is partially financed by

\section{惡CNPq}

da Ciência e Tecnologia

Institutional Sponsors
DFAPESP
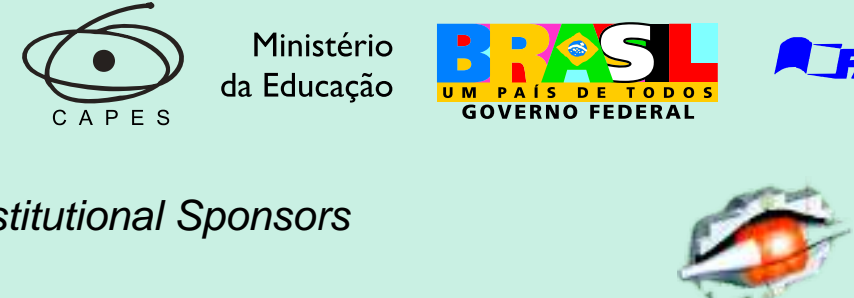

$\sin \theta_{0}$
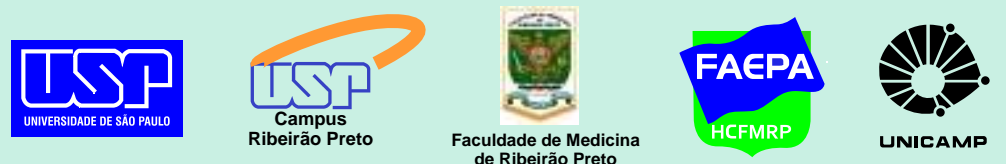

๑ SHIMADZU

Explore High - Performance MS In Protrap Technology

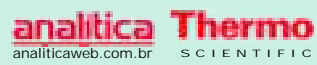




\title{
Effect of gender on training-induced vascular remodeling in SHR
}

\author{
S.L. Amaral1,3 and L.C. Michelini²
'Departamento de Educação Física, Faculdade de Ciências,
Universidade Estadual Paulista “Júlio de Mesquita Filho", Bauru, SP, Brasil
2Departamento de Fisiologia e Biofísica, Instituto de Ciências Biomédicas, Universidade de São Paulo,
São Paulo, SP, Brasil \\ Universidade Federal de São Carlos, São Carlos, SP, Brasil
}

\begin{abstract}
There is accumulating evidence that physical inactivity, associated with the modern sedentary lifestyle, is a major determinant of hypertension. It represents the most important modifiable risk factor for cardiovascular diseases, which are the leading cause of morbidity and mortality for both men and women. In addition to involving sympathetic overactivity that alters hemodynamic parameters, hypertension is accompanied by several abnormalities in the skeletal muscle circulation including vessel rarefaction and increased arteriole wall-to-lumen ratio, which contribute to increased total peripheral resistance. Low-intensity aerobic training is a promising tool for the prevention, treatment and control of high blood pressure, but its efficacy may differ between men and women and between male and female animals. This review focuses on peripheral training-induced adaptations that contribute to a blood pressure-lowering effect, with special attention to differential responses in male and female spontaneously hypertensive rats (SHR). Heart, diaphragm and skeletal muscle arterioles (but not kidney arterioles) undergo eutrophic outward remodeling in trained male SHR, which contributed to a reduction of peripheral resistance and to a pressure fall. In contrast, trained female SHR showed no change in arteriole wall-to-lumen ratio and no pressure fall. On the other hand, training-induced adaptive changes in capillaries and venules (increased density) were similar in male and female SHR, supporting a similar hyperemic response to exercise.
\end{abstract}

Key words: Exercise training; Hypertension; Microcirculation; Arterioles; Capillaries; Venules

\section{Introduction}

In this review, we focus on peripheral mechanisms to explain hemodynamic changes in male and female hypertensive individuals submitted to exercise training. For a better understanding we first review the mechanisms that condition cardiovascular adjustments in hypertension and then discuss several adaptive peripheral mechanisms that trigger a training-induced pressure fall. It is important to note that exercise training is also very effective in causing central adjustments (neuronal plasticity, excitability changes, etc.) of ascending/descending pathways that integrate bulbar and hypothalamic areas involved in cardiovascular control. For a comprehensive understanding of the central effects on autonomic pathways in both normotensive and hypertensive individuals the reader is referred to other excellent papers and reviews (1-13).

\section{Mechanisms conditioning cardiovascular changes in hypertension}

It is well known that, independent of the etiology, the chronic phase of hypertension is maintained mainly by increases in total peripheral resistance, with cardiac output close to normal values (14). It has also been proposed that hypertension is characterized by both overactivity of the sympathetic nervous system that alters vasomotor control and by several abnormalities in tissue microcirculation, such as increased arteriolar wall-to-lumen ratio and decreased vessel density, which together contribute to maintaining an elevated total pe-

Correspondence: L.C. Michelini, Departamento de Fisiologia e Biofísica, Instituto de Ciências Biomédicas, USP, Av. Prof. Lineu Prestes, 1524, 05508-000 São Paulo, SP, Brasil. Fax: +55-11-3091-7285. E-mail: michelin@usp.br

Presented at the XV Simpósio Brasileiro de Fisiologia Cardiovascular, São Paulo, SP, Brazil, February 2-5, 2011.

Received February 7, 2011. Accepted April 13, 2011. Available online May 2, 2011. Published September $16,2011$. 
ripheral resistance (15-21). In spontaneously hypertensive rats (SHR), the best known experimental model for essential or primary hypertension in humans, we confirmed that high blood pressure levels are accompanied by remodeling of arterioles (increased wall-to-lumen ratio) and capillary rarefaction in many tissues including the skeletal muscles, in which we demonstrated a normal resting blood flow, besides the altered vascular structure (22-26).

According to Mulvany (16), vascular remodeling in hypertension is characterized by increased wall-to-lumen ratio of arteries and arterioles, being classified as eutrophic (decrease in both lumen and outer diameter, with unaltered wall thickness or cross-sectional area) or hypertrophic (increase in media thickening, that encroaches on the lumen). Mulvany (16) also postulated that remodeling of small arterioles in SHR is mainly associated with a eutrophic rather than hypertrophic mechanism. Accordingly, we have previously shown that high blood pressure in male SHR is maintained by eutrophic remodeling of arterioles since we observed an increased wall-to-lumen ratio without changes in wall thickness in the kidney, heart, diaphragm, and in locomotor and non-locomotor muscles $(22,25)$. Eutrophic remodeling in the resistance arteries is the most prevalent vascular adjustment to hypertension (20) and could be explained by a combination of growth and apoptotic processes to maintain the media volume (15).

Laurent et al. (18) have also proposed a cross-talk between large and small arteries. They stated that increased wall-to-lumen ratio and rarefaction of small arteries are key factors increasing blood pressure. The increased pressure causes large artery stiffness and determines increased pulse pressure, which consequently damages the small arteries and capillaries. More recently, Cheng et al. (21) have demonstrated that several changes in capillary morphology are associated with elevated systolic and diastolic blood pressure, even in individuals with mild pressure elevations.

Previous data from our laboratory have shown that, although elevated pressure in SHR is caused by an increased arteriolar wall-to-lumen ratio, this process occurs differently in male and female SHR. While male rats exhibited an increased wall-to-lumen ratio of small arterioles in all territories such as skeletal muscles, diaphragm, myocardium, and kidney $(22,25)$, female SHR showed no change in wall-tolumen ratio in many tissues, with a marked increase in the renal arterioles (26). Indeed, Moreno et al. (27) reported a high kidney weight in female hypertensive rats (vs normotensive controls), which could be a useful "intermediate phenotype" of hypertension. These observations agree with pressure measurements obtained by us and by others: male SHR had higher pressure levels than female SHR (range of $170-185$ vs $150-160 \mathrm{mmHg}$ for males and females, respectively) $(22,25,26)$. In general, sex differences have not been extensively explored, since most of the studies were done with male animals. Several mechanisms are proposed to explain gender differences in pressure levels:
1) increased estrogen (28) or decreased testosterone levels $(29,30) ; 2)$ differential activity of the renin-angiotensin system and its association with oxidative stress (29-31); 3 ) gender differences in vasomotor reactivity, which depends on the anatomic origin of the artery (32).

\section{Mechanisms conditioning training-induced hemodynamic changes in SHR}

It is well known that exercise training causes a large improvement in physical capacity in both humans and in animal models. In this regard, we have shown that aerobic training increases running performance on a treadmill, measured as attained velocity, time or distance of running in both normotensive and hypertensive male and female rats $(24-26,33,34)$. Although previous aerobic capacity differs between strains (SHR exhibited a better performance than age-matched WKY normotensive controls) and genders (compared to males, females attained higher velocities during maximal exercise tests on treadmill), we have shown that 3 months of low-intensity aerobic training (50-60\% of maximal exercise capacity) induced similar increases in treadmill performance, independent of strain and/or gender (24-26,33,34).

Aerobic training is currently recommended as a potent coadjuvant of the pharmacological treatment of hypertension, mainly because it attenuates (but does not normalize) resting blood pressure in hypertensive animals $(2,22,23,25,34-39)$ and humans $(40,41)$.

\section{Effects of training on blood pressure levels}

The mechanism(s) underlying blood pressure reduction is (are) still controversial. A fall in blood pressure was shown to be associated with several factors such as decreased vasomotor sympathetic activity $(9,38)$, reduced insulin resistance (42), decreased plasma volume and smaller cardiac output (35), small vascular reactivity (43), reduced total peripheral resistance $(22,25)$, decreased activity of the renin-angiotensin system, and reduced oxidative stress $(34,44)$. It has also been shown that an exercise-induced fall in pressure was associated with an altered balance between relaxing and contractile endothelium-derived factors, causing an improvement of endothelial function (32) and induction of anti-apoptotic genes (45).

We have demonstrated that male SHR submitted to 3 months of low-intensity aerobic training during the established phase of hypertension exhibited an $8-10 \%$ fall in mean arterial pressure (MAP, Figure 1) (22,23,25,33,34,36-38). These results agree with those reported by other authors who have evaluated the effects of exercise training in hypertensive humans $(40,41,46,47)$ and animals $(2,39)$. We have also shown that a training-induced MAP fall was strongly associated with both remodeling of skeletal muscle arterioles (normalization of wall-to-lumen ratio) and a reduction of rela- 
tive hind limb resistance, which were respectively elevated and significantly high in sedentary male SHR compared to age-matched WKY controls $(22,25)$. Figure 1 illustrates this correlation, showing that a training-induced pressure fall was positively correlated with a decreased hind limb resistance in trained SHR. We also showed that a pressure fall in trained SHR was accompanied by normalization of arteriolar wall-to-lumen ratio in several tissues such as locomotor and non-locomotor skeletal muscles, myocardium and diaphragm (Figure 2). The training-induced structural remodeling of these tissues (eutrophic outward remodeling according to Mulvany's classification) caused, as shown in Figure 1, a reduction of local resistance, thus contributing to both the decrease of total peripheral resistance and to the $8-10 \%$ pressure fall. It should be noted that pressure was not normalized in trained SHR because the arterioles of non-exercised tissues (renal and splanchnic territories, for example, see Figure 2) remained hypertrophied, thus maintaining a still elevated total peripheral resistance $(22,25)$. In the WKY control group there were no traininginduced changes in arteriolar wall-to-lumen ratio of any tissue, no resistance changes and therefore no pressure fall (Figures 1 and 2).

Exercise training was not effective in reducing MAP in age-matched female SHR submitted to a similar training protocol $(24,26)$. Interestingly, as observed in Figure 3, in sedentary female SHR hypertension was accompanied by a very strong hypertrophy of the kidney arterioles (wall-tolumen ratio was 2 to 2.5 times greater than that observed in male SHR; compare Figures 3 and 2), but not in the skeletal muscles or heart arterioles that presented a wall-to-lumen ratio in the same range as that of male and female normotensive controls (26). There was no hypertrophy to be reversed by training in skeletal muscle or heart arterioles; therefore, training did not change local resistance in exercised tissues of female SHR. As commented above, training was unable to change the wall-to-lumen ratio of non-exercised tissues, and therefore there was no reduction in total peripheral resistance and no pressure fall $(24,26)$. Recently, Schlüter et al. (39) published an elegant meta-analysis confirming that sex, age and exercise duration do interfere with the blood pressure response to exercise. It was concluded
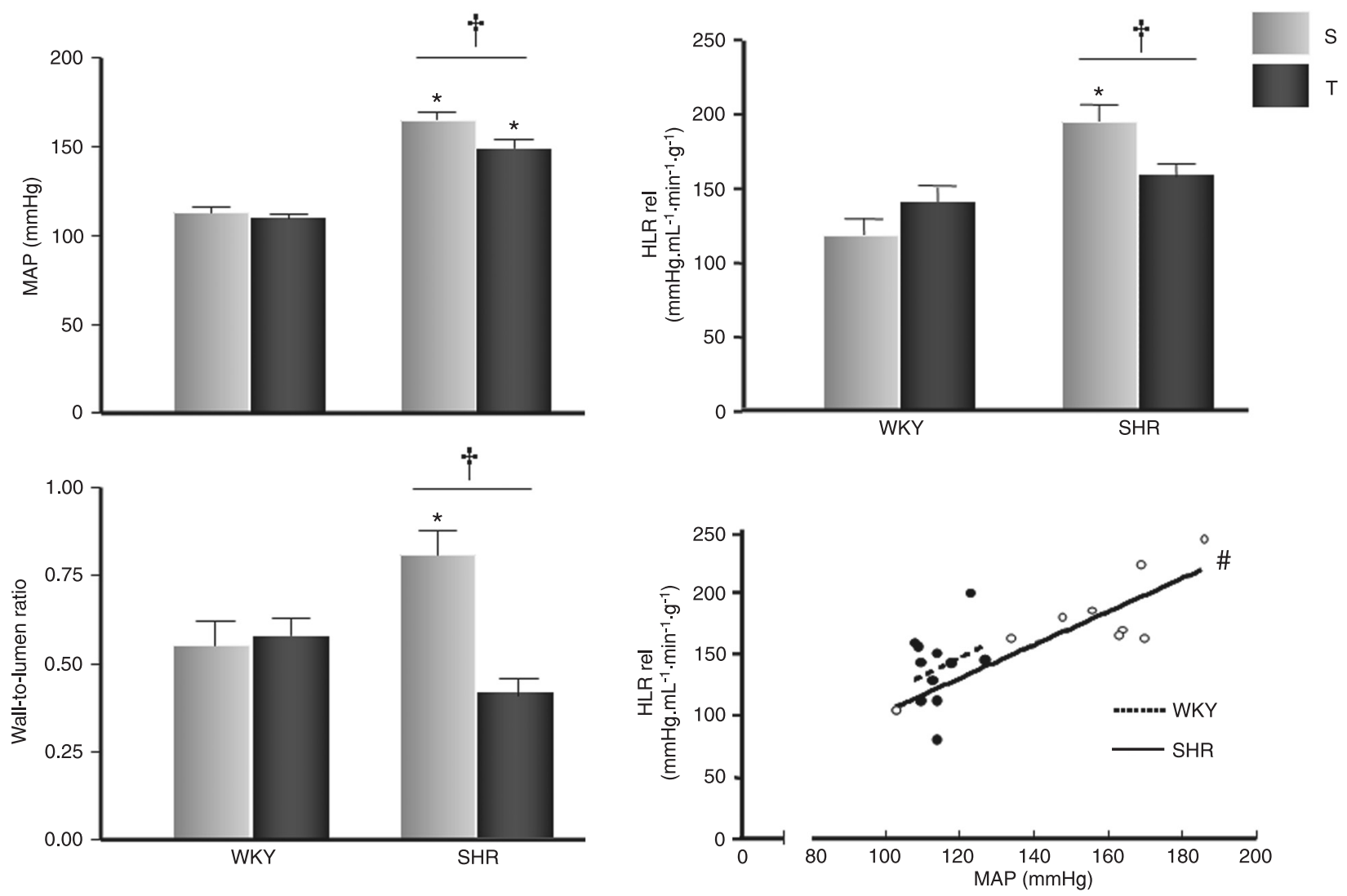

Figure 1. Resting values of mean arterial pressure (MAP); relative hind limb resistance (HLR rel) and arteriolar wall-to-lumen ratio in the gracilis muscle of sedentary $(\mathrm{S})$ and trained $(\mathrm{T})$, normotensive (WKY) and spontaneously hypertensive (SHR) rats at rest. Nine to 12 rats/group. * $\mathrm{P}<0.05$ vs WKY; $\uparrow \mathrm{P}<0.05$ vs $\mathrm{S}$ (two-factor ANOVA). Lower right panel, Correlation between HLR rel and respective MAP in sedentary/trained WKY and SHR groups. Linear regression equations are: $Y_{W K Y}=1.46 x-28, r=0.28(P>0.05)$; $Y_{S H R}=1.35 x$ - 32, $r=0.83(P<0.05)$ : \#denotes a significant correlation (Pearson correlation test). Adapted from Ref. 22, with permission. 
that 2-month-old SHR submitted to 3 months of treadmill training presented a differential response of systolic blood pressure: a 14-mmHg reduction in males vs $1-\mathrm{mmHg}$ fall in females. These investigators also concluded that the blood pressure-lowering effect was higher when training started during the pre-hypertensive phase or at an early stage, being in this case independent of exercise duration. Considering only young rats in the hypertensive stage, it was also shown that prolonged exercise protocols caused a greater pressure reduction (39). These results confirmed several previous observations by our group regarding the effectiveness of exercise training in reducing blood pressure in males but not in females (22-26). Contrary to data reported by Schlüter et al. (39), we were also able to show that older SHR trained for up to 3 months may receive some benefits from exercise training, including a small but significant pressure reduction accompanied by a significant reduction of arteriolar wall-to-lumen ratio in locomotor muscles (Caffaro RR, Oliveira RAF, Amaral SL, Baldo MVC, Michelini LC, Rossoni LV, unpublished data).

\section{Training effects on the heart: heart rate and cardiac hypertrophy}

In addition to a pressure fall, a heart rate reduction is also present in male and female SHR, in other animal models as well as in human hypertension (2,3,5,25,26,34,36-41). Indeed, resting bradycardia is an important marker of exercise training. Several mechanisms could contribute to this response. Some studies have shown that exercise training causes a significant reduction of intrinsic heart rate (48). Martins et al. (36) and Higa-Taniguchi et al. (37) also demonstrated that training-induced resting bradycardia was accompanied by increased oxytocin mRNA expression and increased oxytocin content within the dorsal brainstem (including the nucleus tractus solitarii, NTS) and the dorsal
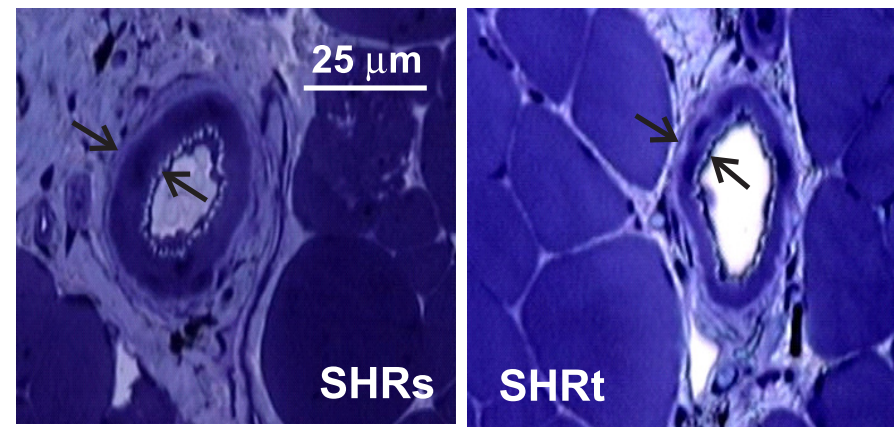

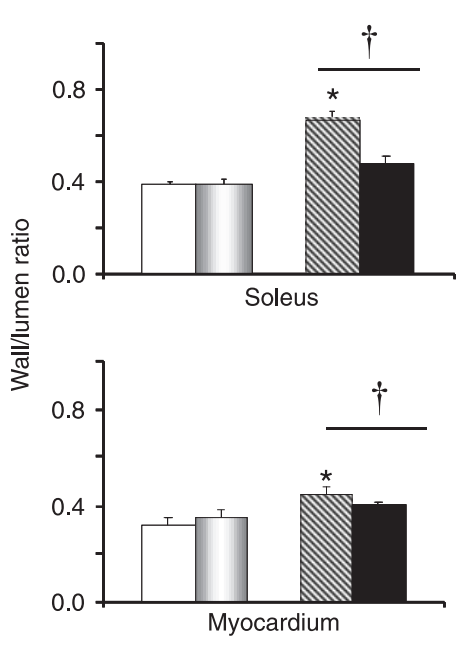

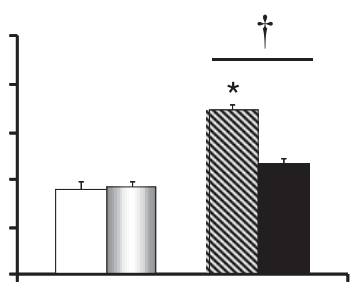

Gracilis

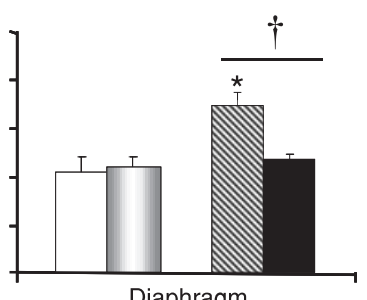

Diaphragm

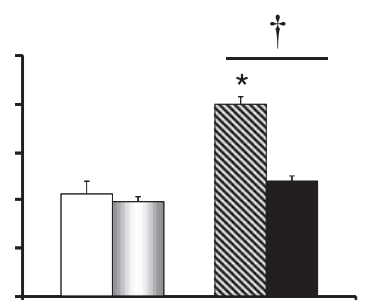

Gastrocnemius

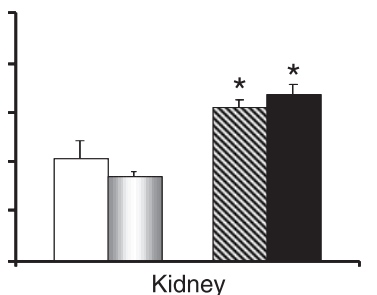

Kidney

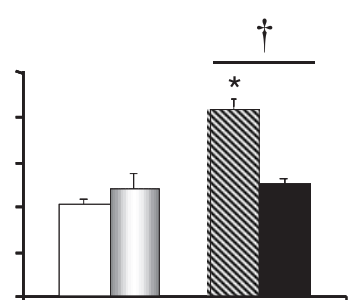

Temporalis

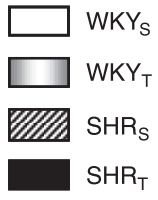

Figure 2. Upper panels, Photomicrographs taken from transverse sections of gracilis muscle arterioles of sedentary (s, left panel) and trained (t, right panel) spontaneously hypertensive (SHR) male rats. Arrows indicate wall thickness; bar = $25 \mathrm{~mm}$. Lower panels, Effects of hypertension and training on arteriolar wall/lumen ratio in different tissues of sedentary (S) and trained (T) normotensive (WKY) and SHR male rats. Mean values correspond to 11-40 arterioles in the myocardium, 9-18 in the kidney and 5-9 arterioles in the skeletal muscles taken from 3-4 rats per group. *P $<0.05$ vs WKY; ${ }^{\dagger} \mathrm{P}<0.05$ vs S (two-factor ANOVA). Adapted from Ref. 25, with permission. 
motor nucleus of the vagus, DMV) of both WKY and SHR groups. In previous studies, we have already shown that activation of oxytocinergic projections from the paraventricular nucleus (PVN) of the hypothalamus to the NTS-DMV area in trained individuals caused a great improvement of the vagal tonus to the heart, which contributes to the appearance of resting bradycardia in trained WKY and SHR $(10,37)$. Reductions of sympathetic activity to the heart and improvements in baroreceptor reflex control have also been shown to contribute to the lower heart rate after exercise training in hypertensive humans and animals $(3,38,40)$, in such a way that trained hypertensive individuals exhibited reduced sympathetic and increased vagal tonus to the heart while trained normotensive individuals presented a significantly higher vagal outflow to the heart. Schlüter et al. (39) confirmed in their review that resting heart rate is reduced after exercise training irrespective of the age of the rat at the beginning or at the end of the exercise period. Accordingly, we have already shown that low-intensity treadmill exercise training was largely efficient in causing resting bradycardia in both WKY and SHR $(25,33,34,37)$.

In a recent study, we also showed that higher traininginduced activity of PVN oxytocinergic projections to the dorsal brainstem during an acute bout of exercise was also
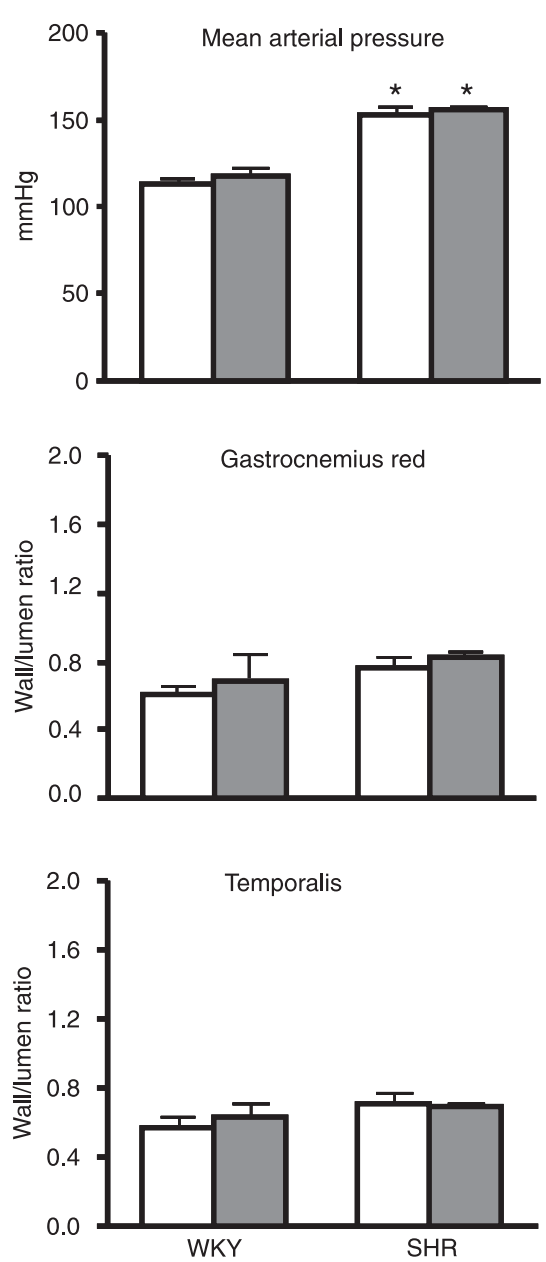
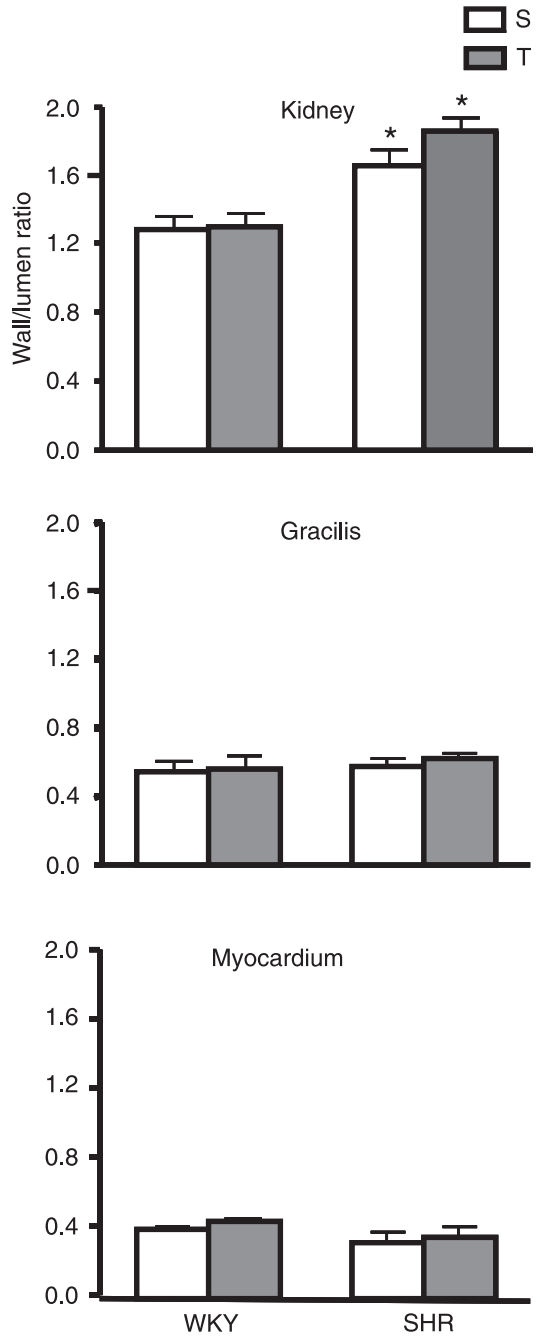
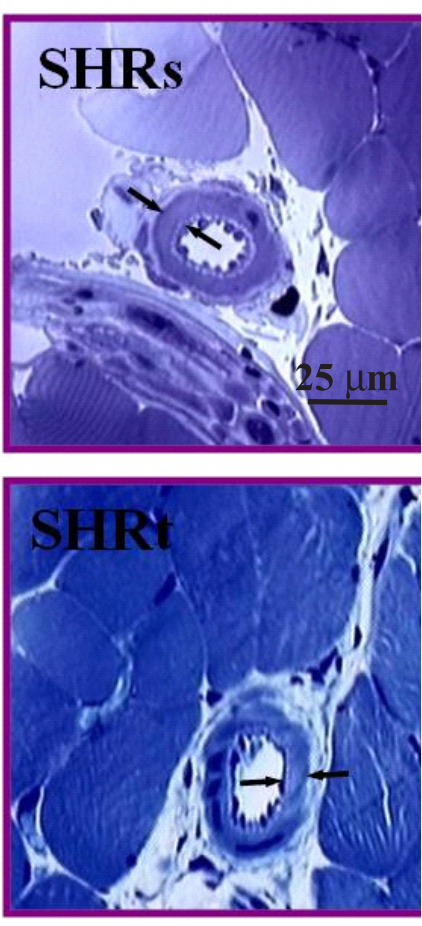

Figure 3. Effects of hypertension and training on resting values of mean arterial pressure and on arteriolar wall-to-lumen ratio in different tissues of sedentary (S) and trained (T) normotensive (WKY) and spontaneously hypertensive (SHR) female rats. Mean values correspond to 20-30 arterioles in the myocardium and kidney and 5-15 arterioles in the skeletal muscles taken from 4-8 rats per group. ${ }^{*} \mathrm{P}<0.05$ vs WKY (two-factor ANOVA). Right panels, Photomicrographs taken from transverse sections of gastrocnemius muscle arterioles of sedentary ( $\mathrm{s}$, upper micrograph) and trained (t, lower micrograph) SHR. Arrows indicate wall thickness; bar $=25 \mathrm{~mm}$. Adapted from Ref. 26, with permission. 
involved in the reduction of exercise tachycardia in both WKY and SHR groups since blockade of oxytocin receptors within the NTS/DMV area caused significant blunting of the tachycardia response (larger in the WKY) in both trained groups (37). Interestingly, oxytocin receptor blockade did not change exercise tachycardia in sedentary WKY and SHR, indicating that increased activity of oxytocinergic PVN-NTS/DMV projections is a specific response of trained normotensive and hypertensive individuals, also being a marker of training.

Concentric cardiac hypertrophy is normally present in hypertension and can be defined as an increased heart mass (myocyte hypertrophy), usually involving increased interstitial fibrosis and apoptosis, which lead to cardiac dysfunction (13). Accordingly, many studies have shown an increased left ventricle weight-to-body weight (LV/BW) ratio in male and female $\operatorname{SHR}(22,26,39)$. However, in the compensated phase of hypertension, an increased LV/BW ratio is not associated with cardiac dysfunction in female SHR (26). Regarding the effects of exercise training on the heart, exercise usually causes eccentric hypertrophy. A meta-analysis by Schlüter et al. (39) indicated that lowintensity treadmill training (not free running) was able to decrease the heart weight-to-body weight ratio in SHR, but only in very young rats, mostly in the pre-hypertensive stage. Using SHR at the established phase of hypertension, we were not able to demonstrate a reduction of the LV/BW ratio in trained male (22) and female (26) groups. In their recent review on cardiac hypertrophy and the differences between concentric and eccentric hypertrophy, Bernardo et al. (13) showed that the type of hypertrophy depends on the stimulus (pressure load or volume load) for heart wall remodeling. This comprehensive review demonstrated that endurance exercise hypertrophy, or eccentric hypertrophy is always associated with ventricle enlargement, but with a proportional change in wall thickness in such a way that cardiac function is normal or even enhanced. In this model, there is no fibrosis and hypertrophy is reversible. Therefore, we may assume that aerobic training brings benefits to the heart, promoting an eccentric hypertrophy to improve the ejection fraction and to ameliorate the relaxation dysfunction induced by hypertension (49-51). On the other hand, pathological hypertrophy induced by hypertension, myocardial infarction and dilated cardiomyopathy leads to cardiac dysfunction, fibrosis, necrosis and/or apoptosis of the myocardium (13). There are many candidate genes to explain physiological cardiac hypertrophy. A pathway downstream to $\mathrm{PI} 3 \mathrm{~K}$ and AKT/mTOR has been proposed, which is induced by growth factors, mainly insulin-like growth factor-1 (IGF-1) (13,50).

\section{Effects of training on blood flow}

Since exercise causes a decrease of heart rate and a stroke volume increase, it was assumed that cardiac out- put does not change or changed very little after training. During an acute bout of exercise, however, both heart rate and stroke volume increased, with a significant increase in cardiac output in WKY and SHR groups, accompanied by blood flow redistribution with a larger increase in flow to skeletal muscle and a reduced flow to the kidney and splanchnic circulations (52). Indeed, the exercise-induced increase of blood flow in exercised tissues (proportional to exercise intensity) is one of the main circulatory effects occurring during aerobic exercise. The redistribution of cardiac output is responsible for maintaining an adequate perfusion to exercised skeletal muscles in which the metabolic needs are enormously increased. Measuring hind limb flow by the transit-time ultrasonic technique in rats at rest and during an acute bout of exercise we were able to reproduce this phenomenon (53). Figure 4 illustrates that at the beginning of exercise (even at mild exercise of 0.4 $\mathrm{km} / \mathrm{h}$ ) there was a large and prompt increase in hind limb blood flow (vasodilatation of the iliac artery) that attained a steady-state plateau between the 1 st and 2 nd min of exercise. Increases in exercise intensity were accompanied by a further increase in hind limb flow as observed when the load was changed from 0.4 to $0.8 \mathrm{~km} / \mathrm{h}$ (moderate exercise intensity). On the other hand, the same exercise protocol caused a mild blood flow decrease in the renal artery at 0.4 $\mathrm{km} / \mathrm{h}$ but a significant flow reduction (marked vasoconstriction) when exercise intensity was changed from 0.4 to 0.8 $\mathrm{km} / \mathrm{h}$. As observed in Figure 4, blood flow redistribution is accompanied by marked tachycardia ( +100 to $+150 \mathrm{bpm}$, proportional to exercise intensity), but also by a small blood pressure raise $(+10$ to $+15 \mathrm{mmHg})$, which is maintained throughout the exercise.

A study from our laboratory compared pressure, heart rate, hind limb flow, and local resistance changes at rest and during mild to moderate exercise in sedentary and trained male WKY and SHR (23). Resting pressure differed between groups, but pressure responses to graded exercise were quite similar: at the onset of exercise there was a prompt pressure increase (slightly higher in trained SHR) that was maintained during the exercise bout (Figure 5). The heart rate response was higher in SHR but it developed gradually and was proportional to exercise intensity in all groups. Figure 5 also illustrates a huge exercise-induced increase in hind limb flow during exercise (exercise hyperemia) in both sedentary and trained WKY and SHR. The mechanisms controlling blood flow during exercise are still controversial. Removal of a sympathetic vasoconstrictor (responsible for maintaining resting vascular tone) (54), accumulation of local metabolic vasodilators such as adenosine, nitric oxide, osmolarity, high $\mathrm{CO}_{2}$, low $\mathrm{O}_{2}$ and $\mathrm{pH}(52,54)$ and high activity of the muscle pump (55) are proposed mechanisms.

As observed in Figure 5, the onset of exercise was characterized by a quick flow increment that was similar in all groups during the first minutes. However, at higher 
intensities (performed only by the trained groups) the relative flow increment was higher in trained SHR than in trained WKY. One also observed that flow increases during the onset of exercise were caused by prompt vasodilatation occurring at different degrees: in sedentary SHR the initial flow increase was attained with a marked decrease in local resistance while in the trained SHR it was achieved with a small vasodilatation, similar to that presented by WKY groups at mild to moderate exercise intensities. Therefore, in all groups the instantaneous initial vasodilatation (occurring in the first 1-4 s) is mainly caused by removal of sympathetic vasomotor tone (56). The exercise-induced reduction of resistance in the trained SHR was progressive, with maximal vasodilatation being attained at higher intensities (Figure $5)$ : at $1.4 \mathrm{~km} / \mathrm{h}$ the reduction of resistance in the trained SHR was similar to that observed early in the sedentary SHR and did not differ from that presented by trained WKY. Late vasodilatation is mostly mediated by accumulation
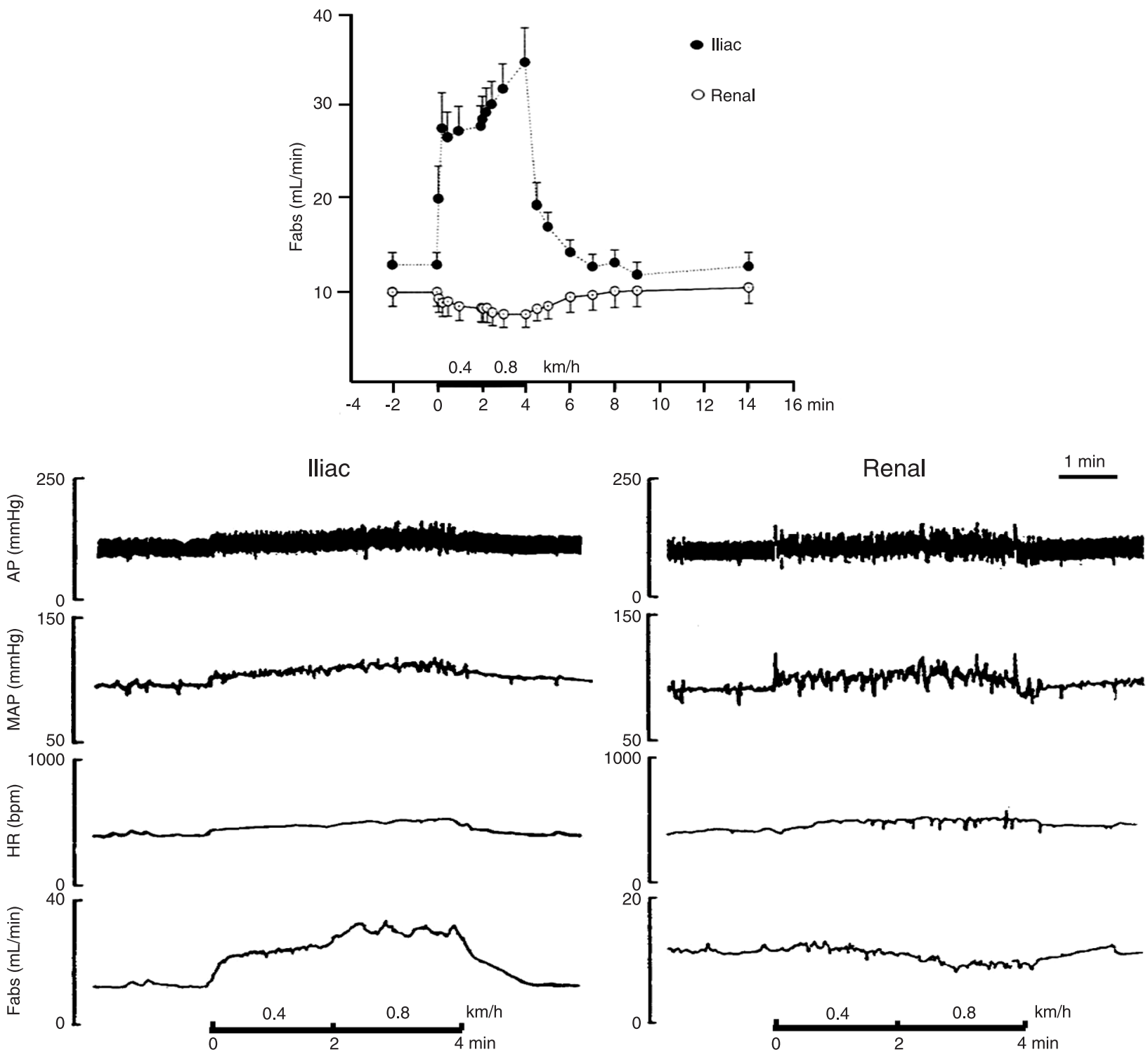

Figure 4. Upper panel, Changes in absolute regional flow (Fabs) in two different tissues: iliac $(N=7)$ and renal $(N=10)$ at rest, during 4 min of exercise (black bar) at 2 different intensities and during recovery. Lower panels, Recordings of pulsatile (AP) and mean arterial pressure (MAP), heart rate (HR) and mean regional absolute flow (Fabs) for 2 rats with a chronically implanted 1-mm probe (left panel, iliac flow and right panel, renal flow) during rest, mild $(0.4 \mathrm{~km} / \mathrm{h})$ to moderate $(0.8 \mathrm{~km} / \mathrm{h})$ exercise intensity (black bars) and recovery. Reproduced from Ref. 53, with permission. 
of local vasoactive compounds, which act directly on the vessel wall and indirectly to block sympathetic activation (functional sympatholysis). Indeed several investigators have confirmed that sympathetic vasomotor innervation is activated during exercise $(52,54-56)$. Despite the similar local vasodilatation occurring in both groups, relative flow at high exercise load was significantly higher in trained SHR than in trained WKY (Figure 5), suggesting the involvement of other factor(s).

As shown in Figure 6, low-intensity training is effective in causing angiogenesis in all exercised tissues $(22,25)$ : capillary density was markedly increased in all locomotor muscles, in the heart and diaphragm of both trained WKY and trained SHR, without significant changes in non-locomotor (temporalis) and renal tissues. The larger capillary supply in skeletal muscles, myocardium and diaphragm reflects the increased oxygen uptake in exercise-activated tissues, being an adaptive local response to augmented flow during exercise. It has been shown that exercise-induced shear-stress is one of the possible mechanisms explaining angiogenesis, mainly by activation of nitric oxide, vascular endothelial growth factor (VEGF) and VEGF receptor (57). In agreement, we have demonstrated that VEGF has a key role in physiological angiogenesis (58). Greater capillary density could contribute to maintaining an increased flow in trained individuals, but it does not explain the differential changes in flow during graded exercise in trained SHR and trained WKY because it was similarly increased in both groups (Figure 6) $(22,25)$.

Differential changes in flow during graded exercise could be explained by adaptive venule responses induced by training. Previous studies from our laboratory have shown a marked venular growth in trained SHR, specific for small venules from 8-10 to 40-45 $\mu \mathrm{m}$ in inner diameter, which indicates vessel neoformation (Figure 7). Interestingly training-induced venular growth is specific for the skeletal muscle circulation (locomotor and non-locomotor), and does not appear in other exercised tissues such as
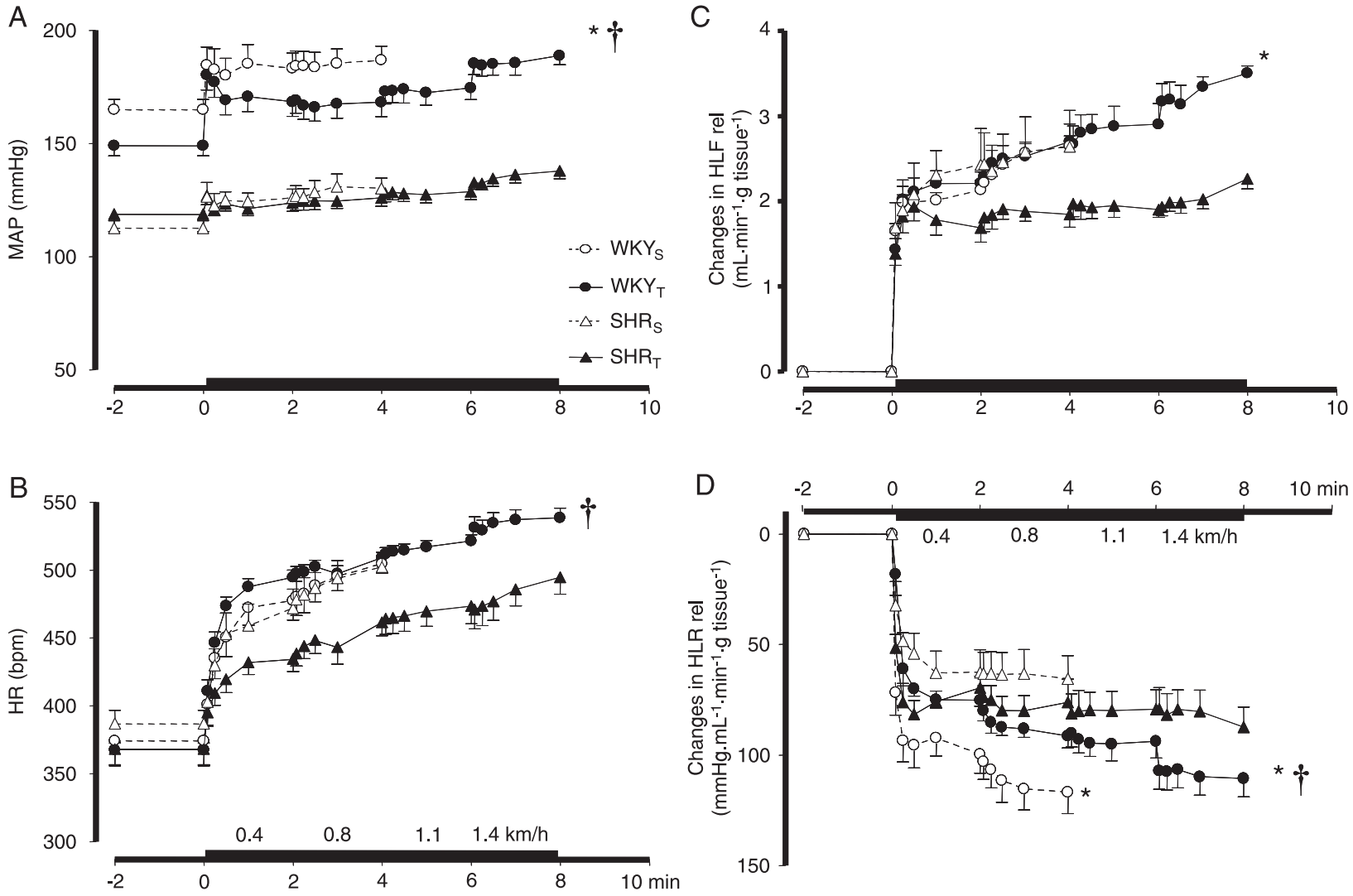

Figure 5. Left panels, Absolute values of mean arterial pressure (MAP, A) and heart rate (HR, B) at rest and during graded exercise (black bar) of sedentary (S) and trained (T) normotensive (WKY) and spontaneously hypertensive (SHR) rats. Right panels, Changes in relative hind limb flow (HLF rel, C) and relative hind limb resistance (HLR rel, D) during graded exercise (black bar) in sedentary (S) and trained (T) WKY and SHR. Exercise intensities are $0.4,0.8,1.1$, and $1.4 \mathrm{~km} / \mathrm{h}, 2 \mathrm{~min}$ each. Six to 12 rats/group. ${ }^{*} \mathrm{P}<0.05$ vs WKY; tP $<0.05$ vs S (two-factor ANOVA). Adapted from Ref. 23, with permission. 

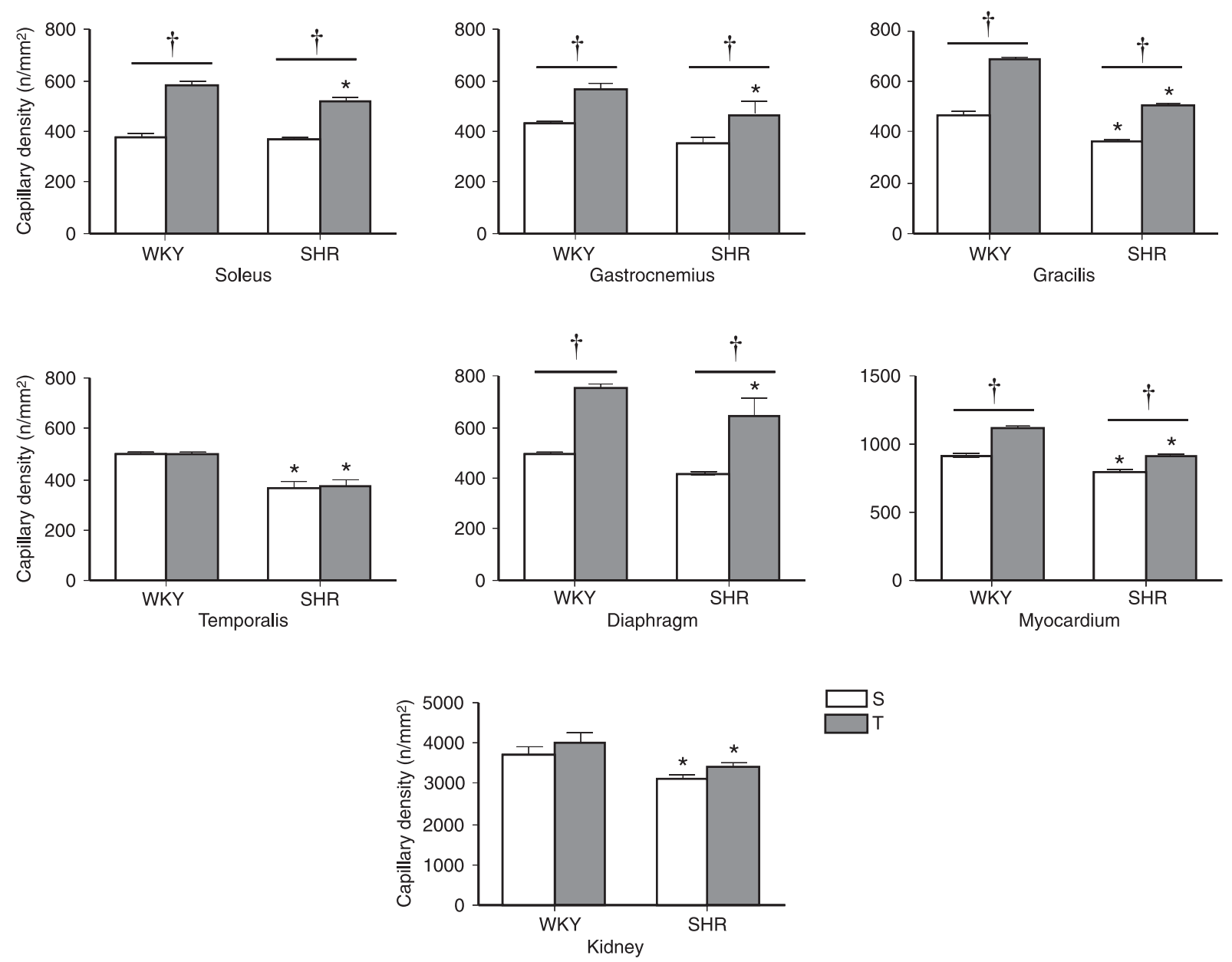

Figure 6. Comparison of capillary density $\left(\mathrm{n} / \mathrm{mm}^{2}\right)$ in exercised (soleus, gastrocnemius, gracilis, diaphragm, and myocardium) and non-exercised tissues (temporalis and kidney) of sedentary ( $\mathrm{S}$, open columns) and trained ( $\mathrm{T}$, filled columns) male normotensive (WKY) and spontaneously hypertensive (SHR) rats. Values are from 9-12 slices taken from 3-4 rats per group. ${ }^{*} \mathrm{P}<0.05$ vs WKY; ${ }^{\dagger} \mathrm{P}$ $<0.05$ vs S (two-factor ANOVA).

the myocardium and diaphragm $(22,25)$. This specificity supports the participation of the muscle pump, also known as the venous pump, in flow adjustments during exercise (59). In fact, large venule conductance in the active skeletal muscles supports high pumping activity during exercise. It is important to note that training-induced venular growth is also specific for SHR, without any change in trained WKY (Figure 7). By increasing venous capacitance in an already large tissue (veins contain $\sim 70 \%$ of the total blood volume), it contributes to reducing blood volume/vascular capacity and to accommodating the larger muscle inflow during exercise hyperemia. This is an interesting adjustment in trained SHR: increased venular bed, besides contributing to the reduction of the mean circulatory filling pressure (which is increased in several models of hypertension) (14), accommodates a large venular endothelial surface favoring the production/release of endothelium-derived factors, which by reaching adjacent arterioles can affect vascular tone and thus the local flow (60). In a previous study (23), we also demonstrate that the greater exercise hyperemia in the trained SHR is positively correlated with increased venule density in locomotor muscles. As shown in the lower panel of Figure 7, the hind limb flow increase was higher in trained SHR rats, which exhibited an increased venular bed; there was no correlation in the WKY group in which there was no training-induced venular growth.

In order to elucidate possible gender discrepancies in exercise hyperemia, we compared local flow and adaptive capillary/venule responses within skeletal muscles in male and female SHR (26). Besides the absence of a pressure fall in trained female SHR (which is explained by the lack of remodeling in skeletal muscle arterioles; compare Figures 1-3 to Figure 8), resting hind limb resistance was lower in sedentary female SHR compared to male sedentary SHR, 

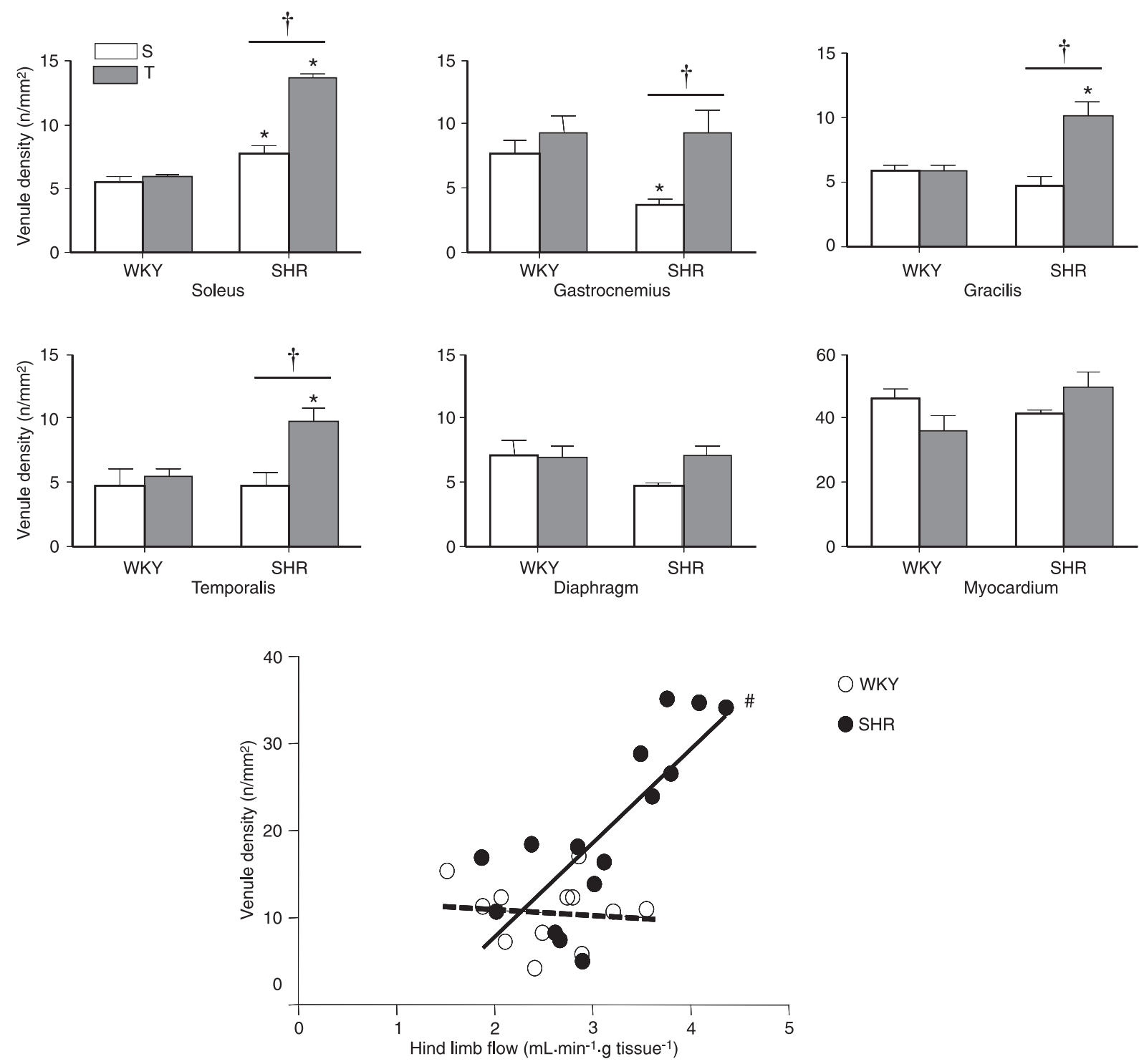

WKY

SHR

Figure 7. Upper panels, Comparison of venule density $\left(\mathrm{n} / \mathrm{mm}^{2}\right)$ in diaphragm, myocardium and skeletal muscles (soleus, gastrocnemius, gracilis, and temporalis) of sedentary (S) and trained (T) male normotensive (WKY) and spontaneously hypertensive (SHR) rats. Values are from 9-12 slices taken from 3-4 rats per group. ${ }^{*} \mathrm{P}<0.05$ vs WKY; ${ }^{\dagger} \mathrm{P}<0.05$ vs $\mathrm{S}$ (two-factor ANOVA). Lower panel, Correlation between relative hind limb flow and respective venule density in the gracilis muscle of sedentary/trained WKY and SHR groups. Linear regression equations are: $Y_{W K Y}=-0.57 x+12, r=-0.09(P>0.05) ; Y_{S H R}=10.79 x-14, r=0.78(P<0.05)$; ${ }^{\text {denotes a }}$ significant correlation for SHR data (Pearson correlation test).

thus causing an elevated hind limb flow at rest. In female SHR, training was accompanied by a significant increase in capillary/fiber ratio and a huge increase in venule density (Figure 8) (26). These responses (which were similar to those presented by trained male SHR) combined with an already reduced skeletal muscle resistance cause a large increment in vascular conductance at rest. In order to keep a near normal blood flow at rest, trained female SHR exhibited a significant increase in resting hind limb resistance to compensate for the high conductance. On the other hand, during an acute bout of exercise sympathetic vasoconstriction was withdrawn, allowing a large blood inflow in trained female SHR (26). It should be noted that exercise hyperemia in female SHR (data not shown) was similar to that observed in males and was explained by the similar adaptive responses of venule density within the skeletal muscles.

In conclusion, different training-induced adaptive responses in the skeletal muscle arterioles of male (eutrophic outward remodeling) and female (no change) SHR support 

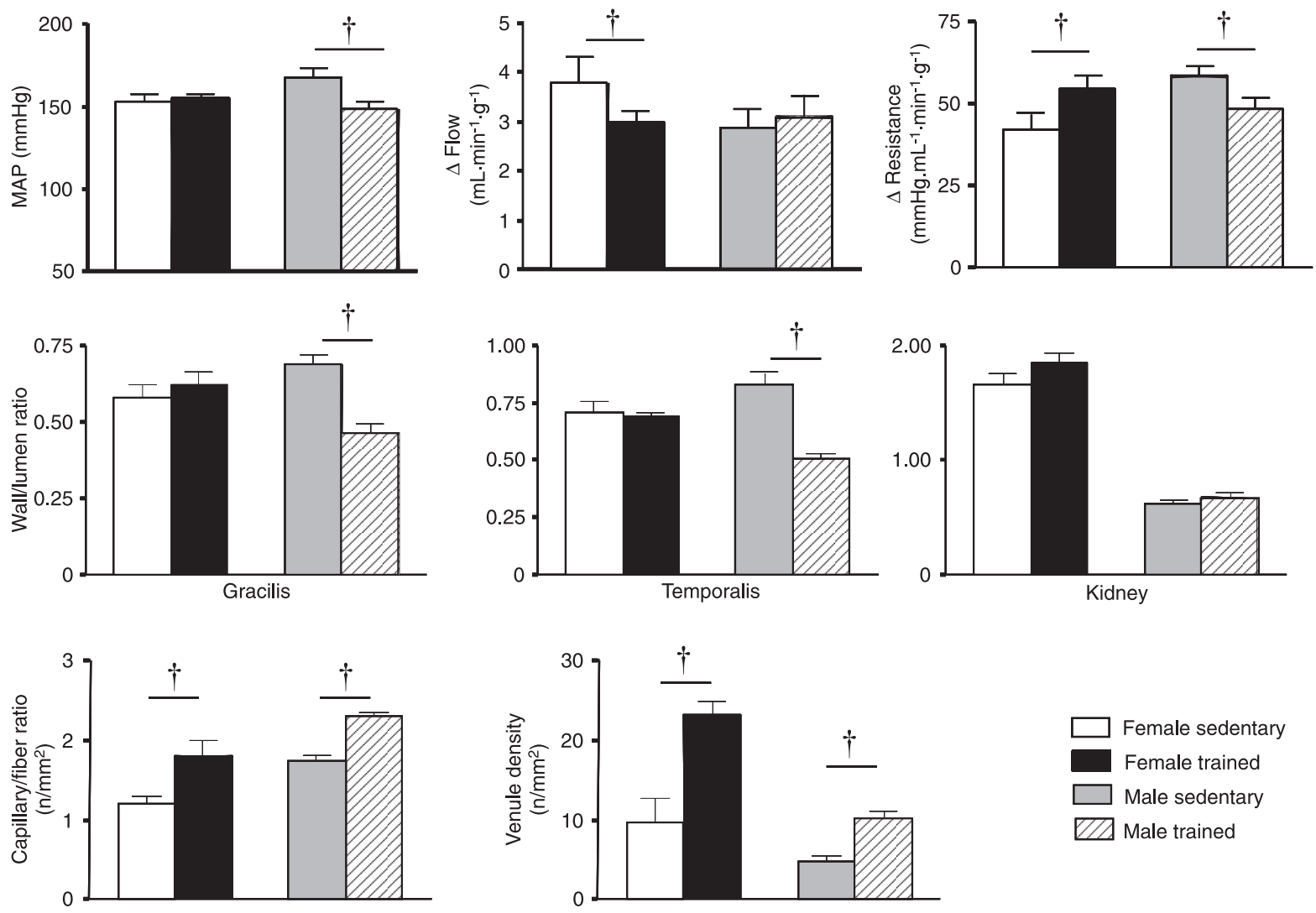

emale sedentary

Female trained

Male sedentary

$\measuredangle$ Male trained

Figure 8. Comparison of hemodynamic (mean arterial pressure, MAP), hind limb flow and resistance; upper panels) and vascular adaptations in arterioles (wall-to-lumen ratio in the gracilis, temporalis and kidney; middle pane/s), capillaries and venules (density values in locomotor skeletal muscles; lower panels) of male and female spontaneously hypertensive rats submitted to low-intensity aerobic training. Eleven to 12 rats/group. ${ }^{\dagger} \mathrm{P}<0.05$ vs $\mathrm{S}$ (two-factor ANOVA). Adapted from Ref. 26, with permission.

the differential pressure response: a moderate fall in male SHR and no pressure change in female SHR submitted to exercise training. On the other hand, the training-induced venular growth observed in both trained male and female SHR conditions a similar hyperemic response to exercise.

\section{References}

1. Takeshita A, Jingu S, Imaizumi $T$, Kunihiko $Y$, Koyanagi $S$, Nakamura M. Augmented cardiopulmonary baroreflex control of forearm vascular resistance in young athletes. Circ Res 1986; 59: 43-48.

2. Brum PC, Da Silva GJ, Moreira ED, Ida F, Negrao CE, Krieger EM. Exercise training increases baroreceptor gain sensitivity in normal and hypertensive rats. Hypertension 2000; 36: 1018-1022.

3. Blumenthal JA, Babyak MA, Hinderliter A, Watkins LL, Craighead L, Lin PH, et al. Effects of the DASH diet alone and in combination with exercise and weight loss on blood pressure and cardiovascular biomarkers in men and women with high

\section{Acknowledgments}

Research supported by CNPq and FAPESP (\#02/05761-5, $\# 02 / 11937-9$ and \#03/01578-4) L.C. Michelini is a CNPq Research Fellow. blood pressure: the ENCORE study. Arch Intern Med 2010; 170: 126-135.

4. O'Sullivan SE, Bell C. The effects of exercise and training on human cardiovascular reflex control. J Auton Nerv Syst 2000; 81: 16-24.

5. Michelini LC. Oxytocin in the NTS. A new modulator of cardiovascular control during exercise. Ann N Y Acad Sci 2001; 940: 206-220.

6. Jackson K, Silva HM, Zhang W, Michelini LC, Stern JE. Exercise training differentially affects intrinsic excitability of autonomic and neuroendocrine neurons in the hypothalamic paraventricular nucleus. J Neurophysiol 2005; 94: 3211- 
3220.

7. Potts JT. Inhibitory neurotransmission in the nucleus tractus solitarii: implications for baroreflex resetting during exercise. Exp Physiol 2006; 91: 59-72.

8. Mueller PJ. Exercise training and sympathetic nervous system activity: evidence for physical activity dependent neural plasticity. Clin Exp Pharmacol Physiol 2007; 34: 377-384.

9. Mueller PJ. Physical (in)activity-dependent alterations at the rostral ventrolateral medulla: influence on sympathetic nervous system regulation. Am J Physiol Regul Integr Comp Physiol 2010; 298: R1468-R1474.

10. Michelini LC. Differential effects of vasopressinergic and oxytocinergic pre-autonomic neurons on circulatory control: reflex mechanisms and changes during exercise. Clin Exp Pharmacol Physiol 2007; 34: 369-376.

11. Michelini LC. The NTS and integration of cardiovascular control during exercise in normotensive and hypertensive individuals. Curr Hypertens Rep 2007; 9: 214-221.

12. Michelini LC, Stern JE. Exercise-induced neuronal plasticity in central autonomic networks: role in cardiovascular control. Exp Physiol 2009; 94: 947-960.

13. Bernardo BC, Weeks KL, Pretorius L, McMullen JR. Molecular distinction between physiological and pathological cardiac hypertrophy: experimental findings and therapeutic strategies. Pharmacol Ther 2010; 128: 191-227.

14. Guyton AC, Hall JE, Coleman TG, Manning RD Jr, Norman RA Jr. The dominant role of the kidneys in long-term arterial pressure regulation in normal and hypertensive states. In: Laragh JH, Brenner BM (Editors), Hypertension: pathophysiology, diagnostic and management. New York: Raven Press; 1995. p 1311-1326.

15. Intengan HD, Schiffrin EL. Structure and mechanical properties of resistance arteries in hypertension: role of adhesion molecules and extracellular matrix determinants. Hypertension 2000; 36: 312-318.

16. Mulvany MJ. Small artery remodeling and significance in the development of hypertension. News Physiol Sci 2002; 17 : 105-109.

17. Laurent $S$, Boutouyrie $P$, Lacolley $P$. Structural and genetic bases of arterial stiffness. Hypertension 2005; 45: 10501055.

18. Laurent S, Briet M, Boutouyrie P. Large and small artery cross-talk and recent morbidity-mortality trials in hypertension. Hypertension 2009; 54: 388-392.

19. Yasmin, O'Shaughnessy KM. Genetics of arterial structure and function: towards new biomarkers for aortic stiffness? Clin Sci 2008; 114: 661-677.

20. Martinez-Lemus LA, Hill MA, Meininger GA. The plastic nature of the vascular wall: a continuum of remodeling events contributing to control of arteriolar diameter and structure. Physiology 2009; 24: 45-57.

21. Cheng C, Daskalakis C, Falkner B. Alterations in capillary morphology are found in mild blood pressure elevation. $J$ Hypertens 2010; 28: 2258-2266.

22. Amaral SL, Zorn TM, Michelini LC. Exercise training normalizes wall-to-lumen ratio of the gracilis muscle arterioles and reduces pressure in spontaneously hypertensive rats. J Hypertens 2000; 18: 1563-1572.

23. Amaral SL, Silveira NP, Zorn TM, Michelini LC. Exercise training causes skeletal muscle venular growth and alters hemodynamic responses in spontaneously hypertensive rats. J Hypertens 2001; 19: 931-940.
24. Amaral SL, Sanchez LS, Chang AJ, Rossoni LV, Michelini LC. Time course of training-induced microcirculatory changes and of vegf expression in skeletal muscles of spontaneously hypertensive female rats. Braz J Med Biol Res 2008; 41: 424-431.

25. Melo RM, Martinho E Jr, Michelini LC. Training-induced, pressure-lowering effect in SHR: wide effects on circulatory profile of exercised and nonexercised muscles. Hypertension 2003; 42: 851-857.

26. Coimbra R, Sanchez LS, Potenza JM, Rossoni LV, Amaral SL, Michelini LC. Is gender crucial for cardiovascular adjustments induced by exercise training in female spontaneously hypertensive rats? Hypertension 2008; 52: 514-521.

27. Moreno C, Dumas P, Kaldunski ML, Tonellato PJ, Greene AS, Roman RJ, et al. Genomic map of cardiovascular phenotypes of hypertension in female Dahl S rats. Physiol Genomics 2003; 15: 243-257.

28. Dubey RK, Oparil S, Imthurn B, Jackson EK. Sex hormones and hypertension. Cardiovasc Res 2002; 53: 688-708.

29. Lopez-Ruiz A, Sartori-Valinotti J, Yanes LL, Iliescu R, Reckelhoff JF. Sex differences in control of blood pressure: role of oxidative stress in hypertension in females. Am J Physiol Heart Circ Physiol 2008; 295: H466-H474.

30. Yanes LL, Sartori-Valinotti JC, Iliescu R, Romero DG, Racusen LC, Zhang $\mathrm{H}$, et al. Testosterone-dependent hypertension and upregulation of intrarenal angiotensinogen in Dahl salt-sensitive rats. Am J Physiol Renal Physiol 2009; 296: F771-F779.

31. Sartori-Valinotti JC, Iliescu R, Yanes LL, Dorsett-Martin W, Reckelhoff JF. Sex differences in the pressor response to angiotensin II when the endogenous renin-angiotensin system is blocked. Hypertension 2008; 51: 1170-1176.

32. Laughlin $M H$, Schrage WG, McAllister RM, Garverick HA, Jones AW. Interaction of gender and exercise training: vasomotor reactivity of porcine skeletal muscle arteries. J Appl Physiol 2001; 90: 216-227.

33. Higa-Taniguchi KT, Silva FC, Silva HM, Michelini LC, Stern JE. Exercise training-induced remodeling of paraventricular nucleus (nor)adrenergic innervation in normotensive and hypertensive rats. Am J Physiol Regul Integr Comp Physiol 2007; 292: R1717-R1727.

34. Felix JV, Michelini LC. Training-induced pressure fall in spontaneously hypertensive rats is associated with reduced angiotensinogen mRNA expression within the nucleus tractus solitarii. Hypertension 2007; 50: 780-785.

35. Veras-Silva AS, Mattos KC, Gava NS, Brum PC, Negrao CE, Krieger EM. Low-intensity exercise training decreases cardiac output and hypertension in spontaneously hypertensive rats. Am J Physiol 1997; 273: H2627-H2631.

36. Martins AS, Crescenzi A, Stern JE, Bordin S, Michelini LC. Hypertension and exercise training differentially affect oxytocin and oxytocin receptor expression in the brain. Hypertension 2005; 46: 1004-1009.

37. Higa-Taniguchi KT, Felix JV, Michelini LC. Brainstem oxytocinergic modulation of heart rate control in rats: effects of hypertension and exercise training. Exp Physiol 2009; 94: 1103-1113.

38. Ceroni A, Chaar LJ, Bombein RL, Michelini LC. Chronic absence of baroreceptor inputs prevents training-induced cardiovascular adjustments in normotensive and spontaneously hypertensive rats. Exp Physiol 2009; 94: 630-640.

39. Schlüter KD, Schreckenberg $R$, da Costa Rebelo RM. Inter- 
action between exercise and hypertension in spontaneously hypertensive rats: a meta-analysis of experimental studies. Hypertens Res 2010; 33: 1155-1161.

40. Laterza MC, de Matos LD, Trombetta IC, Braga AM, Roveda $\mathrm{F}$, Alves MJ, et al. Exercise training restores baroreflex sensitivity in never-treated hypertensive patients. Hypertension 2007; 49: 1298-1306.

41. Cardoso CG Jr, Gomides RS, Queiroz AC, Pinto LG, da Silveira LF, Tinucci T, et al. Acute and chronic effects of aerobic and resistance exercise on ambulatory blood pressure. Clinics 2010; 65: 317-325.

42. Chen YL, Liu YF, Huang CY, Lee SD, Chan YS, Chen CC, et al. Normalization effect of sports training on blood pressure in hypertensives. J Sports Sci 2010; 28: 361-367.

43. Pasqualini L, Schillaci G, Innocente S, Pucci G, Coscia F, Siepi $D$, et al. Lifestyle intervention improves microvascular reactivity and increases serum adiponectin in overweight hypertensive patients. Nutr Metab Cardiovasc Dis 2010; 20: 87-92.

44. Pialoux V, Brown AD, Leigh R, Friedenreich CM, Poulin MJ. Effect of cardiorespiratory fitness on vascular regulation and oxidative stress in postmenopausal women. Hypertension 2009; 54: 1014-1020.

45. Watson PA, Reusch JE, McCune SA, Leinwand LA, Luckey $\mathrm{SW}$, Konhilas JP, et al. Restoration of CREB function is linked to completion and stabilization of adaptive cardiac hypertrophy in response to exercise. Am J Physiol Heart Circ Physiol 2007; 293: H246-H259.

46. Cicero AF, Derosa G, Bove M, Di Gregori V, Gaddi AV, Borghi C. Effect of a sequential training programme on inflammatory, prothrombotic and vascular remodelling biomarkers in hypertensive overweight patients with or without metabolic syndrome. Eur J Cardiovasc Prev Rehabil 2009; 16: 698704.

47. Hansen AH, Nielsen JJ, Saltin B, Hellsten Y. Exercise training normalizes skeletal muscle vascular endothelial growth factor levels in patients with essential hypertension. $\mathrm{J} \mathrm{Hy}$ pertens 2010; 28: 1176-1185.
48. Boyett MR. 'And the beat goes on'. The cardiac conduction system: the wiring system of the heart. Exp Physiol 2009; 94: 1035-1049.

49. Goodman JM, Liu PP, Green HJ. Left ventricular adaptations following short-term endurance training. J Appl Physiol 2005; 98: 454-460.

50. Glass DJ. Skeletal muscle hypertrophy and atrophy signaling pathways. Int J Biochem Cell Biol 2005; 37: 1974-1984.

51. Cocco G, Pandolfi S. Physical exercise with weight reduction lowers blood pressure and improves abnormal left ventricular relaxation in pharmacologically treated hypertensive patients. J Clin Hypertens 2011; 13: 23-29.

52. Delp MD, O'Leary DS. Integrative control of the skeletal muscle microcirculation in the maintenance of arterial pressure during exercise. J Appl Physiol 2004; 97: 1112-1118.

53. Amaral SL, Michelini LC. Validation of transit-time flowmetry for chronic measurements of regional blood flow in resting and exercising rats. Braz J Med Biol Res 1997; 30: 897908.

54. Thomas GD, Segal SS. Neural control of muscle blood flow during exercise. J Appl Physiol 2004; 97: 731-738.

55. Sarelius I, Pohl U. Control of muscle blood flow during exercise: local factors and integrative mechanisms. Acta Physiol 2010; 199: 349-365.

56. Clifford PS. Skeletal muscle vasodilatation at the onset of exercise. J Physiol 2007; 583: 825-833.

57. Hudlicka O, Brown MD. Adaptation of skeletal muscle microvasculature to increased or decreased blood flow: role of shear stress, nitric oxide and vascular endothelial growth factor. J Vasc Res 2009; 46: 504-512.

58. Amaral SL, Linderman JR, Morse MM, Greene AS. Angiogenesis induced by electrical stimulation is mediated by angiotensin II and VEGF. Microcirculation 2001; 8: 57-67.

59. Sheriff $D$. Point: The muscle pump raises muscle blood flow during locomotion. J Appl Physiol 2005; 99: 371-372.

60. Saito Y, Eraslan A, Lockard V, Hester RL. Role of venular endothelium in control of arteriolar diameter during functional hyperemia. Am J Physiol 1994; 267: H1227-H1231. 